As expected, the doublet $\mathrm{C}++, \mathrm{D}_{3}$ was the hardest to produce with lines of equal intensity; but ultimately a number of quite satisfactory spectra were obtained, on several of which the intermediate line $\mathrm{HeD}$ was visible. The position of this agreed well with the value of the $\mathrm{He}, \mathrm{D}_{2}$ doublet, which is rather higher than that found by Bainbridge.

The following are the most probable values of the isotopic weights on the physical scale :

$$
\begin{aligned}
& \mathrm{H}=1.00812 \pm 0.00004 \\
& \mathrm{D}=2 \cdot 01471 \pm 0.00007 \\
& \mathrm{He}=4.00391 \pm 0.00016 \\
& \mathrm{C}=12.0035 \pm 0.0003 .
\end{aligned}
$$

Cavendish Laboratory,
Cambridge.
Feb. 15.
${ }^{2}$ NATURE, 135, 541 (April 6, 1935).

F. W. Aston.

\section{Cosmic Ray Particles of High Penetrating Power}

I HAVE studied the very penetrating component of cosmic radiation by means of a large vertical Wilson chamber actuated by counters, following the method of Blackett and Occhialini. The chamber is $55 \mathrm{~cm}$. high and $15 \mathrm{~cm}$. broad and is placed between the large poles of $75 \mathrm{~cm}$. diameter of the electromagnet at Bellevue of the Paris Academy of Sciences. The magnetic field is 13,000 gauss over all the chamber. The experimental apparatus is shown in Fig. 1. A $7 \mathrm{~cm}$. block of lead is placed between the counters. Rays such as $A B$ were photographed. The cosmic rays photographed with this arrangement are those which are able to penetrate the lead block, that is, the more penetrative component of the spectrum alone is photographed.

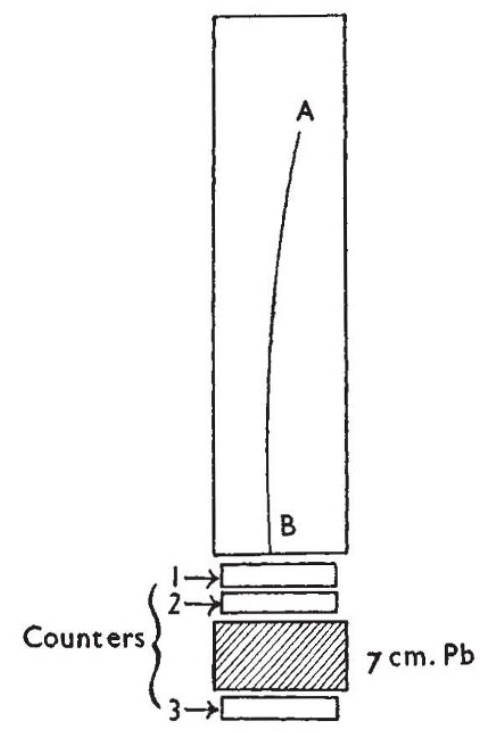

Fig. 1.

To investigate the precision of the measurements, we have taken a number of photographs with no magnetic field and we have measured the displacement of the centre of the track from the straight line joining its ends. We have found that the maximum dis. placement never reached $0.6 \mathrm{~mm}$. for a length of about $40 \mathrm{~cm}$.; the energy corresponding to such a displacement is $12 \times 10^{\circ} \mathrm{e.v}$. Thus we can determine the sign of the particle producing a track $40 \mathrm{~cm}$. long if the energy is not greater than $12 \times 10^{9}$ e.v. The results of the measurements are given in the accompanying table.

\begin{tabular}{|c|c|c|c|}
\hline \multirow{2}{*}{ Energy Range } & \multicolumn{3}{|c|}{ Number of Particles } \\
\cline { 2 - 3 } & +ve & -ve & sign uncertain \\
\hline$>10^{10}$ e.v. & 4 & 0 & 2 \\
$2.5^{\circ} \times 10^{9}$ to $10^{10}$ e.v. & 6 & 1 & 9 \\
$10^{\circ}$ to $2.5 \times 10^{9}$ e.v. & 7 & 6 & 3 \\
\hline
\end{tabular}

In addition to the tracks tested in the table, on positive particle with energy $900 \times 10^{8}$ e.v. was photographed.

(1) We see that for the greatest energies, positive particles are predominant; on the other hand, for the rays of which the energy is less than $2.5 \times 10^{9}$ e.v., there are about as many positive as negative tracks.

(2) The minimum of observed energy is of the same order for particles of both signs: we have observed no ray with an energy lower than $900 \times 10^{6} \mathrm{e} . \mathrm{v}$. This means that a particle of lower energy has but a little probability of crossing the $7 \mathrm{~cm}$. of lead.

These results can be explained by the assumption of two components for cosmic rays, of a different nature: first, negative and positive electrons, some of which are capable of crossing $7 \mathrm{~cm}$. of lead with an energy loss of at least 900 million e.v. This is in agreement with the latest results of Anderson and Neddermeyer ${ }^{1}$ and with the predicted increase of the radiation energy loss with increasing electron energy ${ }^{2}$; secondly, a radiation of a different nature, nearly exclusively composed of positive charged particles, such as protons, which is in agreement with the assumptions of various authors 3,4 .

Louis LEPRINCE-RINGUet.

Laboratoire de Physique des Rayons,

12 rue Lord Byron, Paris VIII'.

'S. H. Neddermeyer and C. D. Anderson, Los Angeles meet., abstr. 32 (1935).

H. Bethe and W. Heitler, Proc. Roy. Soc., A, 856, 83 (1934).

3 A. Compton and H. Bethe, NATURe, 134, 734 (1934).

4. Auger, J. Phys., 7, 226 (1935).

\section{Cosmic Rays and the Origin of Species}

UNDER the above title, Dr. Hamshaw Thomas in a recent paper ${ }^{1}$ has put forward the suggestion that the large variety of species and high percentage of endemics often found at high altitudes might be due to mutants which have been produced by irradiation with cosmic rays, the intensity of which is known to be much higher at great altitudes than at sea-level.

We wish to point out that this hypothesis is some. what invalidated for the following reasons:

(1) It is known ${ }^{2}$ that the rate of mutations induced by X-rays and similar agents (for example, $\beta$ - and $\gamma$-rays of radium) is accurately proportional to the total ionisation per cubic centimetre of irradiated organic material. From this fact one derives without ambiguity the absolute value of the mutation rate induced by cosmic rays at any altitude in any species, if the X-ray induced mutability for this species is known. 